\title{
2010 update on the ROSEBUD project
}

\author{
N. Coron, J. Gironnet, P. de Marcillac, M. Martínez, T. Redon, L. Torres. \\ Institut d'Astrophysique Spatiale (IAS). Bâtiment 121, Université Paris-Sud 11 and CNRS \\ (UMR 8617), 91405 Orsay Cedex, France. \\ E-mail: hoel.corondias.u-psud.fr G.qironnetdipn1.in2p3.fr, \\ pierre.demarcillacdias.u-psud.fr, maria.martinezdias.u-psud.fr \\ thierry.redondias.u-psud.fr, Lidia.torresdias.u-psud.fr
}

C. Cuesta, E. García, C. Ginestra, Y. Ortigoza*, A. Ortiz de Solórzano, C. Pobes, J. Puimedón, T. Rolón, A. Salinas, M.L. Sarsa, J.A. Villar.

Grupo de Física Nuclear y Astropartículas. Universidad de Zaragoza. C/ Pedro Cerbuna 12, 50009 Zaragoza, Spain.

E-mail: ccuestadunizar.es, edqarciadunizar.es, coinestradunizar.es,

ortigozadunizar.es, alfortizdunizar.es, cpobesdunizar.es,

puimedondunizar.es, aroloncdunizares, salinasdunizar.es,

mlsarsadunizar.es, villaraunizar.es

The ROSEBUD (Rare Objects SEarch with Bolometers UndergrounD) Collaboration has tested scintillating bolometers of $\mathrm{BGO}\left(\mathrm{Bi}_{4} \mathrm{Ge}_{3} \mathrm{O}_{12}\right), \mathrm{Al}_{2} \mathrm{O}_{3}$ and $\mathrm{LiF}$ in a common experimental set-up in an ultralow background environment at the Canfranc Underground Laboratory. We present the new results of a recent analysis of the data obtained. The BGO heat and light response to different particles has been measured, focusing on its capability for nuclear recoils discrimination against the $\beta / \gamma$ background. A comparison of BGO sensitivity with that of other targets used or proposed in direct dark matter searches is discussed. We also have analyzed the simultaneous use of LiF and $\mathrm{Al}_{2} \mathrm{O}_{3}$ scintillating bolometers to monitor the fast neutron flux inside the experimental shielding. Monte Carlo simulations of the recoils induced in $\mathrm{Al}_{2} \mathrm{O}_{3}$ by fast neutrons are presented.

Identification of Dark Matter 2010

July 26 - 30, 2010

University of Montpellier 2, Montpellier, France

\footnotetext{
*Speaker.
} 


\section{Introduction}

ROSEBUD is a collaboration between the Institut d'Astrophysique Spatiale (IAS) from Orsay-France and the University of Zaragoza from Spain dedicated to the development and characterization of scintillating bolometers for their use in Nuclear Physics and Astroparticle Physics. Presently, it focuses on dark matter direct detection searches by analyzing the possible implementation of scintillating bolometers in the future EURECA experiment [四]: a 1 ton cryogenic hybrid experiment allowing for different targets.

Our scintillating bolometers consist of a scintillating crystal (the absorber) and facing to it an optical bolometer (a Ge disk). They are mounted inside a copper frame with internal reflecting cavity of Ag and thermally coupled to the mixing chamber of a dilution refrigerator working at $20 \mathrm{mK}$. The energy deposited into the crystal by a particle interaction is converted into heat and light: the heat is measured as a temperature increase of the crystal, and the scintillating photons that escape from the crystal and are absorbed by the optical detector are converted into heat and also measured as a temperature increase. These temperature increases are measured with the respective NTD (neutron transmutation doped) Ge thermistors glued on to the scintillating crystal and to the Ge disk. The simultaneous measurement of heat and light signals (see, for instance, figure $\mathbb{W}$ ) allows us to discriminate among the different particles interacting with the crystal: for the same deposited energy, photons and electrons produce more light than $\alpha$ particles, and $\alpha$ particles more than nuclear recoils, whereas all of them practically produce the same response in heat.

The design, development and preliminary characterization of scintillating bolometers (when low background environment is not needed) is carried out at IAS whereas their characterization in an ultralow background environment is carried out at the Canfranc Underground Laboratory (LSC) under a rock overburden of 2450 meters of water equivalent. In this work we present recent results obtained with three scintillating bolometers of $\mathrm{Al}_{2} \mathrm{O}_{3}(50 \mathrm{~g})$, BGO (46 g) and $\mathrm{LiF}$ $(33 \mathrm{~g})$ characterized for dark matter experiments and/or neutron monitoring. We report on the BGO scintillating bolometer discrimination capability for nuclear recoils against the $\beta / \gamma$ background and a comparison of the sensitivity of BGO and other targets for dark matter direct detection through WIMPs elastic scattering off nuclei. The neutron spectroscopy study performed with $\mathrm{Al}_{2} \mathrm{O}_{3}$ and $\mathrm{LiF}$ scintillating bolometers is also discussed. For further details about the experimental set-up and previous results, see for instance [ $[\square-$ 田].

\section{The $46 \mathrm{~g}$ BGO scintillating bolometer}

The BGO discrimination capability for nuclear recoils against the $\beta / \gamma$ background has been studied in detail at IAS using a ${ }^{252} \mathrm{Cf}$ source and an $\alpha$ source of ${ }^{241} \mathrm{Am}$ which emits ${ }^{237} \mathrm{~Np}$ nuclei. Using the spontaneous fission neutron source of ${ }^{252} \mathrm{Cf}$ we estimated the mean electron versus nuclear recoils relative efficiency factor (REF) for the light signal produced in the BGO, obtaining a mean value of $12.33 \pm 0.57$ [ [ ] ]. With the recoiling ${ }^{237} \mathrm{~Np}$ nuclei from the ${ }^{241} \mathrm{Am}$ source (kinetic energy $92.4 \pm 0.1 \mathrm{keV}$ ) we have estimated a nuclear versus electron recoils thermal REF of $0.937 \pm 0.015_{\text {stat }}+0.004$ syst [焑]. The light response for ${ }^{237} \mathrm{~Np}$ recoiling nuclei and recoils induced by neutrons has been studied using the irradiation with the two sources mentioned above. Figure 四 shows the light versus heat discrimination plot obtained for the exposition to the ${ }^{241} \mathrm{Am}$ source, 


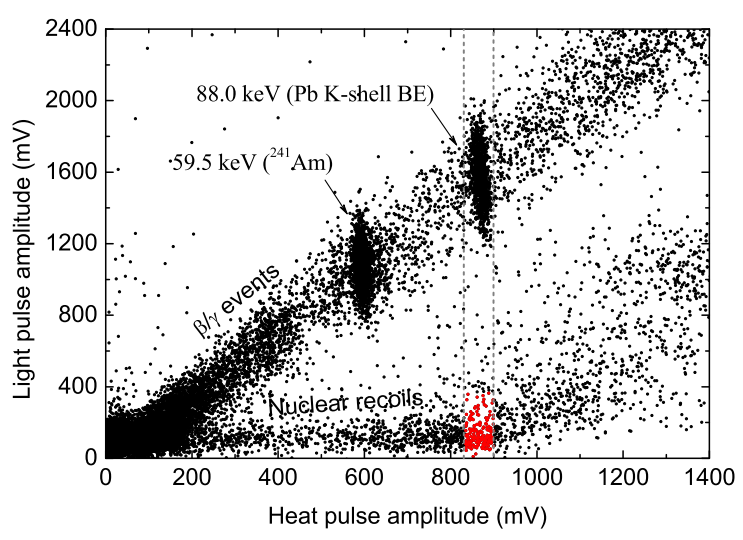

(a)

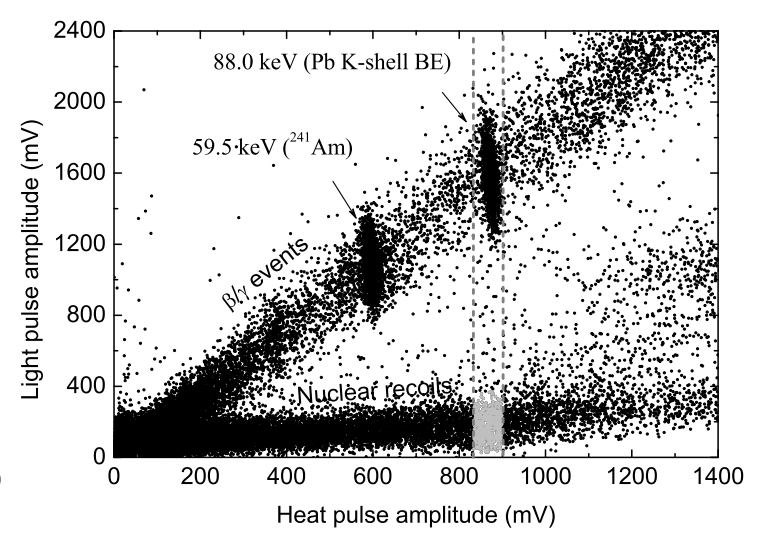

(b)

Figure 1: Light versus heat signal plots obtained with a $46 \mathrm{~g}$ BGO crystal in a calibration with (a) a ${ }^{241} \mathrm{Am}$ source, and (b) with the ${ }^{241} \mathrm{Am}$ and a neutron source of ${ }^{252} \mathrm{Cf}$. Nuclear recoils events (feeble scintillation response) observed in (a) are due to ${ }^{237} \mathrm{~Np}$ recoiling nuclei, whereas those observed in (b) are due to ${ }^{237} \mathrm{~Np}$ recoiling nuclei and recoils of $\mathrm{Bi}, \mathrm{Ge}$ and $\mathrm{O}$ nuclei induced by neutrons. Light distribution of nuclear recoil events obtained in both cases in the heat signal window of $834-900 \mathrm{mV}$ (88 keV events) is shown in figure 2.

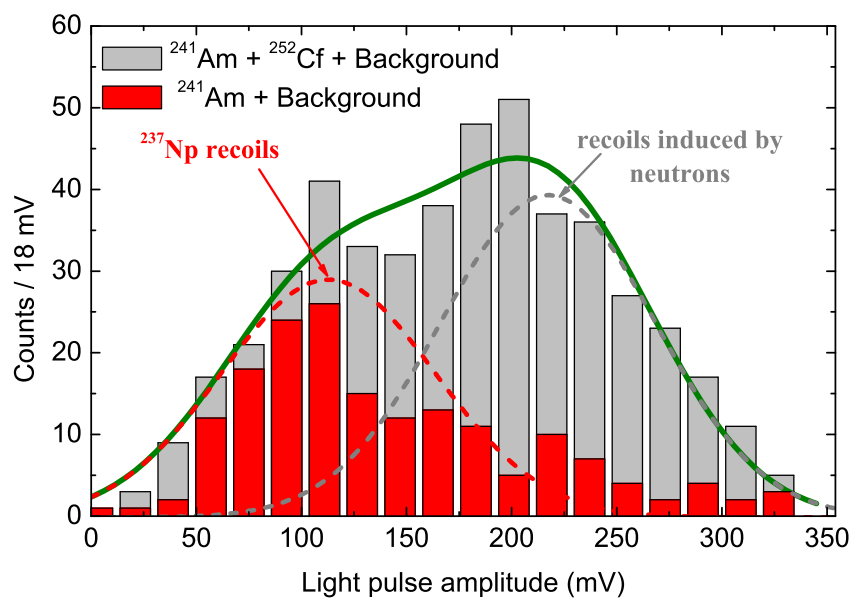

Figure 2: Light distribution of nuclear recoils obtained in the heat signal window 834-900 mV when only the ${ }^{241} \mathrm{Am}$ source was present (red bars) and when we exposed the BGO crystal simultaneously to ${ }^{241} \mathrm{Am}$ and ${ }^{252} \mathrm{Cf}$ sources. A fit to a double Gaussian distribution has been done and, as is explained in the text, they can be ascribed to recoils of ${ }^{237} \mathrm{~Np}$ (the lower one) and to oxygen recoils induced by neutrons (the higher one).

and figure $\square$ b shows the results obtained after the simultaneous exposition to both sources. From the comparison of the figures we can conclude that nuclear recoils induced by neutrons produce a light signal slightly greater than ${ }^{237} \mathrm{~Np}$ recoiling nuclei. This dependence of the light response on the recoiling nuclei mass is more evident in figure $\square$ which shows the light distribution of events recorded in the heat signal window of $88.0 \mathrm{keV}$ ( $\mathrm{Pb} \mathrm{K}$-shell Binding Energy) in both measurements 
colored in figure $\mathrm{W}$. It allows us to identify a double distribution and because the higher amplitude distribution disappears when the ${ }^{252} \mathrm{Cf}$ source is removed, we ascribe this distribution to recoils induced by neutrons and the lower one to ${ }^{237} \mathrm{~Np}$ recoils.

With the mean values derived for these two distributions we have estimated the electron versus nuclear recoils light REF for nuclear recoils induced by neutrons (at this energy, for kinematic reasons, they are practically all oxygen recoils), and for ${ }^{237} \mathrm{~Np}$ recoils obtaining, respectively, $10.5 \pm 0.9$ and $39.5 \pm 15.5$. These light REFs allow us to estimate three nuclear recoils acceptance bands in BGO (see figure (1)): with the mean value of $12.33 \pm 0.57$ one can select nuclear recoils compatible with events induced in any nuclei of the BGO; with the value 10.5, events compatible with oxygen recoils, and with the value 39.5 , events compatible with $\mathrm{Bi}$ (whose mass is similar to that of the Np nucleus) recoils. This selection method improves sensitivity for dark matter searches allowing tuning the search to WIMP masses.

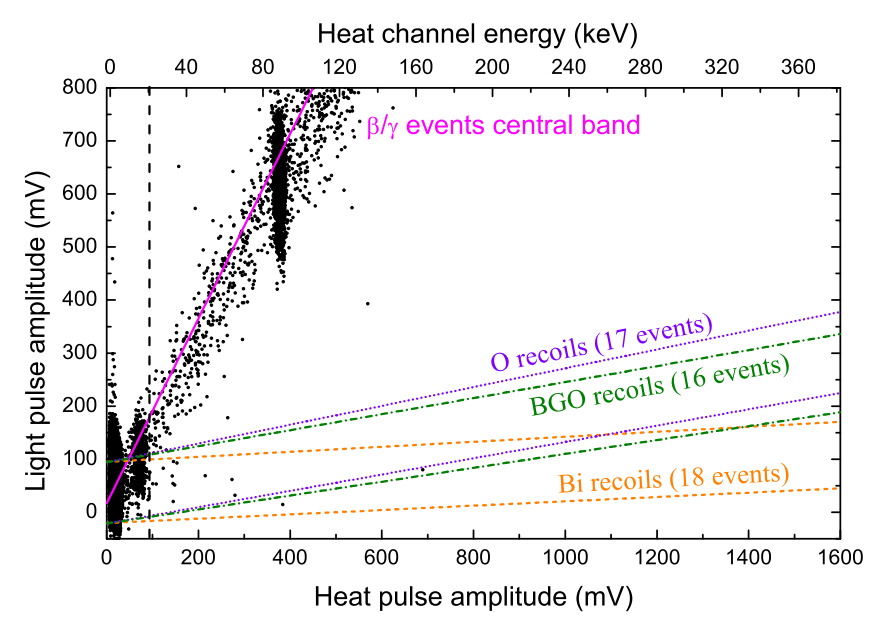

Figure 3: Nuclear recoils acceptance bands (at 90\% confidence level) in the BGO applied to a background measurement carried out at the LSC with a total live time of $9.15 \mathrm{~h}$. Events selected with these bands are compatible with nuclear recoils of $\mathrm{Bi}, \mathrm{O}$ and any nuclei of the BGO.

\section{Comparison of the BGO sensitivity with other WIMP targets}

In order to highlight the BGO capability for dark matter detection we have compared its sensitivity for spin independent interaction (SI) with that of other targets currently used or proposed for WIMP search experiments (see figure 1 ). This comparison has been performed assuming for all the targets the same experimental conditions in nuclear recoils energy threshold, background level, energy resolution and exposition (target mass $\times$ measurement time).

Due to the high atomic mass of $\mathrm{Bi}$, the BGO is a very competitive target for WIMPs with masses greater than $20 \mathrm{GeV} / \mathrm{c}^{2}$, with a sensitivity similar to that of other heavy targets like $\mathrm{CaWO}_{4}$, $\mathrm{Xe}, \mathrm{ZnWO}_{4}$ and NaI. For light WIMPs, targets like $\mathrm{Al}_{2} \mathrm{O}_{3}$ and LiF are clearly more competitive. The BGO has low sensitivity for spin dependent (SD) interactions because of the low expectation value of the proton group's spin $\left\langle S_{p}\right\rangle$ in $\mathrm{Bi}[\square]$ and the low isotopic abundance in ${ }^{17} \mathrm{O}$ and ${ }^{73} \mathrm{Ge}$. 


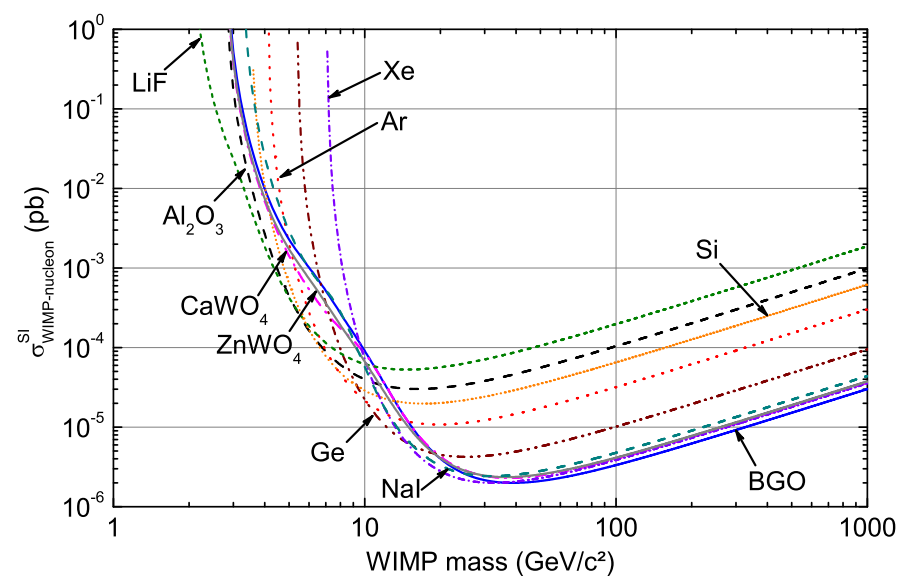

Figure 4: Spin independent exclusion curves at 90\% confidence level obtained for different targets assuming the same experimental conditions: the same coupling strength for WIMPs to protons and neutrons, an energy window $5-10 \mathrm{keV}$ recoil energy, a flat background of $0.1 \mathrm{counts} /(\mathrm{keV} \cdot \mathrm{kg} \cdot \mathrm{day})$, an exposition of $100 \mathrm{~kg}$.day and an energy resolution much better than the window width. Here we can see that the BGO is an interesting target for heavy WIMPs with a sensitivity similar to that of other heavy targets.

\section{Neutron spectroscopy with $\mathrm{LiF}$ and $\mathrm{Al}_{2} \mathrm{O}_{3}$ scintillating bolometers}

We are testing the viability of estimating the fast neutron flux inside the $\mathrm{Pb}$ shielding of the experimental set-up with the simultaneous use of $\mathrm{Al}_{2} \mathrm{O}_{3}$ and $\mathrm{LiF}$ scintillating bolometers. In order to do that, both scintillating bolometers were installed inside a $\mathrm{Pb}$ shielding of $25 \mathrm{~cm}$ in thickness and irradiated with neutrons from a ${ }^{252} \mathrm{Cf}$ source located outside the shielding. In a previous work, we made the hypothesis that the fast neutron flux inside the $\mathrm{Pb}$ shielding could be

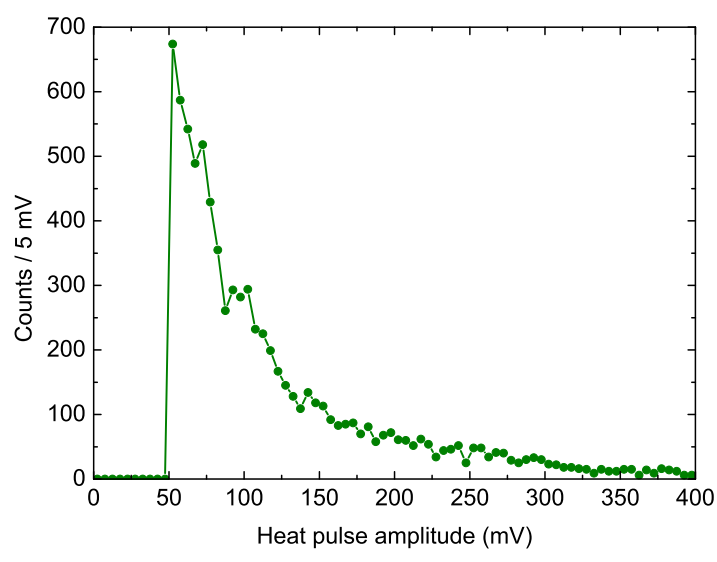

(a)

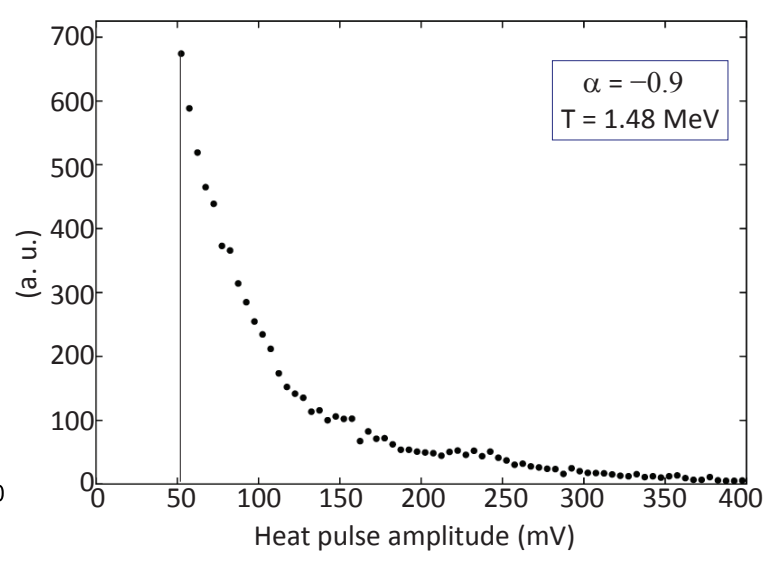

(b)

Figure 5: Sapphire nuclear recoils spectra (a) obtained at the LSC with an exposition to a neutron source of ${ }^{252} \mathrm{Cf}$ located outside the $\mathrm{Pb}$ shielding and (b) simulated with MCNP-PoliMi with the parameters shown in the figure (see text for details). 
described by $\Phi(E) d E=\frac{\Phi_{0}}{\Gamma(\alpha+1)}\left(\frac{E}{T}\right)^{\alpha} e^{-E / T} \frac{d E}{T}$, where $E$ is the neutron energy and $\Phi_{0}, \alpha$ and $T$ are free parameters to be determined. We estimated [ [8] the region of the free parameters $\left(\Phi_{0}, \alpha, T\right)$ compatible with the experimental data using the number of neutron capture events at resonance of ${ }^{6} \mathrm{Li}(n, \alpha)^{3} \mathrm{H}$ and the number of nuclear recoils in $\mathrm{Al}_{2} \mathrm{O}_{3}$ in the interval $[50,100] \mathrm{mV}$ and in the region above $100 \mathrm{mV}$ (shown in figure 5a). The more likely values are $\alpha=-0.9$ and $T=1.48 \mathrm{MeV}$.

In the present work we have tested the hypothesis about the fast neutron flux inside the $\mathrm{Pb}$ shielding profiting from the full shape of the differential $\mathrm{Al}_{2} \mathrm{O}_{3}$ recoils spectrum. To do this, we have performed a Monte Carlo simulation (MCNP-PoliMi [Q] $]$ ) of the neutron flux with the more likely values of the parameters $\alpha$ and $T$, and we have estimated the expected $\mathrm{Al}_{2} \mathrm{O}_{3}$ nuclear recoils spectrum. The simulated spectrum (see figure $5 \mathrm{~b}$ ) obtained is in good agreement with the experimental one, supporting our hypothesis. A full-space parameter comparison is in progress.

\section{Acknowledgments}

This work has been supported by the Spanish Ministerio de Ciencia e Innovación (MICINN) (FPA2008-03228 and MULTIDARK Consolider CSD2009-00064), the French CNRS/INSU (MANOLIA and BOLERO projects) and the EU project ILIAS (RII3-CT-2004-506222). Y. Ortigoza and T. Rolón were also supported by a UZ/BSCH/Fundación Carolina grant. Y. Ortigoza also was supported by a DECRYPT (Detectores de Radiación y Partículas) investigation project contract. L. Torres is supported by a MICINN postdoctoral grant.

\section{References}

[1] H. Kraus et al., EURECA-The future of cryogenic dark matter detection in Europe, EAS Publications Series 36 (2009) 249.

[2] J. Amaré et al., Recent developments on scintillating bolometers for WIMP searches: ROSEBUD status, J. Phys.: Conf. Ser. 39 (2006) 133.

[3] E. García et al., High performance scintillating bolometers in the search for WIMPs: ROSEBUD experiment, in proceedings of The Sixth International Workshop on The Identification of Dark Matter. World Scientific Publishing Co., edited by M. Axenides, G. Fanourakis and J. Vergados. (2007) 302.

[4] N. Coron et al., Sapphire, BGO and LiF scintillating bolometers developed for dark matter experiments, in proceedings of Identification of Dark Matter 2008, POS (idm2008) 007.

[5] N. Coron et al., A BGO scintillating bolometer as dark matter detector prototype, Opt. Mat. 31 (2009) 1393.

[6] N. Coron et al., BGO scintillating bolometer: its application in dark matter experiments, J. Phys.: Conf. Ser. 203 (2010) 012038.

[7] V. Bednyakov and F. Šimkovic, Nuclear spin structure in dark matter search: The zero momentum transfer limit, arXiv:hep-ph/0406218v1, 2004.

[8] N. Coron et al., Detection of fast neutrons with LiF and $\mathrm{Al}_{2} \mathrm{O}_{3}$ scintillating bolometers, J. Phys.: Conf. Ser. 203 (2010) 012139.

[9] S.A. Pozzi, E. Padovani and M. Marseguerra, MCNP-PoliMi: a Monte-Carlo code for correlation measurements, Nucl. Instr. and Meth. A 513 (2003) 550. 